\title{
Etiologic Aspects of Epileptic Seizures of Recent Onset in HIV-AIDS Infected Subjects at the Yaounde Central Hospital (Cameroon)
}

\author{
P. C. Mbonda ${ }^{1 *}$, C. Kuate ${ }^{2,3}$, A. K. Njamnshi ${ }^{2,3}$, Y. Fogang ${ }^{1}$, J. Fonsah ${ }^{2,3}$, W. Muna ${ }^{2,3}$ \\ ${ }^{1}$ Neurology Department, Fann Teaching Hospital, Dakar, Senegal; ${ }^{2}$ Neurology Department, Central Hospital of Yaoundé, University \\ of Yaounde I, Yaounde I, Cameroon; ${ }^{3}$ Faculty of Medicine and Biomedical Sciences, University of Yaounde I, Yaounde I, Cameroon. \\ Email: ${ }^{*}$ mondachimi@yahoo.fr
}

Received February $23^{\text {rd }}, 2013$; revised March 25 $5^{\text {th }}, 2013$; accepted April $2^{\text {nd }}, 2013$

Copyright (C) 2013 P. C. Mbonda et al. This is an open access article distributed under the Creative Commons Attribution License, which permits unrestricted use, distribution, and reproduction in any medium, provided the original work is properly cited.

\begin{abstract}
Introduction: Patients infected with HIV usually develop neurological complications. Seizures are amongst clinical manifestation of these neurological complications of HIV infection. We report on 150 HIV positive patients who presented with newly onset seizures. Objectives: Our objective was to evaluate the influence of HIV infection on epilepsy and particularly to look for the most common epilepsy induced complications in our context. Methodology: We recruited all patients infected with HIV and who consulted at the outpatient or admitted in the in the neurology unit of the Yaounde Central Hospital, for seizures from August 1st 2008 to December 31st 2009. All the patients were aged above 18 years, black and of Cameroonian nationality. All those who had a family history of epilepsy, as well as those with a personal history of epilepsy were excluded from the study. Results: During the period of study, we recruited 150 patients aged $38.7 \pm 9.9$ years and with an $\mathrm{M} / \mathrm{F}$ sex ratio of 0.76 . Generalized seizures were observed in $66 \%$ of patients, of whom $58 \%$ were HIV 1 positive and an average CD4 count of $65.4 \pm 11.7 / \mu$ l. Cerebral toxoplasmosis was the most common etiology observed in 30\% of cases. Discussion: This study being hospital based must have selected the most serious patient with severe immunodeficiency. This can explain why a precise etiology was found in the majority of cases. Modi et al. (1999) found a space occupying lesion in 53\%, meningitis in $22 \%$ and $25 \%$ without identifiable etiology in black South Africans. Central nervous system tuberculosis was the most frequent cause encountered in $64 \%$ of patients with etiologies. Conclusion: Seizures are one of the principal manifestations of HIV infection or its complications. Their prevalence is $9.5 \%$ in our study. Cerebral toxoplasmosis is the most frequent etiology in our study, followed by cryptococcal meningitis and tuberculous meningitis.
\end{abstract}

Keywords: Epilepsy; HIV/AIDS Infection; Cerebral Toxoplasmosis—-Yaoundé (Cameroon)

\section{Introduction}

The central and peripheral nervous system seems to be the site of predilection for the Human Immunodeficiency Virus (HIV). In fact Ogun [1] in a Nigerian study estimated that $45.5 \%$ of patients infected with HIV develop neuronlogical complications and Bhigjee [2] in South Africa estimated a rate of $70 \%$. The neurological complications could be due to the HIV itself, to opportunistic infections or a central nervous system (CNS) lymphoma [3]. Patients infected with HIV usually develop neurological complications like: cerebral toxoplasmosis, tuberculous meningo-enkephalitis, cryptococcal meningitis, cytomegalovirus infection, lymphomas, dementia, myelopathies and

\footnotetext{
${ }^{*}$ Corresponding author.
}

polyneuropathies etc. Seizures are frequently a common clinical manifestation of these pathologies: $3 \%$ in Spain [4], $5 \%$ in India [5], $11 \%$ in the USA [6], $3 \%$ to $17 \%$ in South Africa [2]. Bartholomei et al. [7] in France discovered that $33 \%$ of patients infected by the HIV present with epileptic crisis as inaugural neurological manifestation.

Few studies have evaluated the role of HIV infection on epilepsy in Sub Saharan Africa.

In this study, our objective was to investigate the characteristics of HIV-infected patients with seizures or epilepsy, and determine the etiologies.

\section{Patients and Methods}

We carried out a study of $150 \mathrm{HIV}$-infected patients with 
new onset seizures at the neurology department of the Yaounde Central Hospital in Cameroon from January 2001 to June 2008 for the retrospective phase and from July to December 2009 for the prospective phase. All our patients were above the age of 18 years, black, and of Cameroonian nationality.

All those who had a family history of epilepsy, past history of seizure or a known neurological pathology were excluded from the study (we were only interested in symptomatic seizure from lesions secondary to HIV infection). For all patients we collected sociological, professional and demographical data; the clinical and paraclinical data, as well as etiologic and evolutive data were also reviewed. All patients had a positive HIV serology before or during admission. The type of virus was indicated for each patient in the prospective phase. Many patients in the retrospective phase did not have the type of virus mentioned in their medical files.

Brain CT scan with and without contrast was done in all eligible patients looking for a space occupying lesion (SOL). The diagnosis of brain abscess was made on the basis of one or more hypodense images on the CT scan with ring-like contrast enhancement. Cerebral toxoplasmosis was suspected in the presence of clinical signs of raised intracranial pressure, focal neurological signs, CT scan images of brain abscess and a good clinical and radiological response after 7 - 10 days of anti toxoplasmosis treatment. The diagnosis of tuberculoma was retained in case of failure of treatment for cerebral toxoplasmosis for 10 days, and a favorable outcome to anti tuberculosis. Cerebral lymphoma was considered in the presence of focal neurological signs, signs of raised intracranial pressure, a hypodense image with contrast enhancement and poor therapeutic response to treatment of toxoplasmosis, antituberculosis and antifungal.

In the absence of raised intracranial pressure, a lumbar puncture was performed with CSF analysis (cytology, biochemistry, pathology, bacteriology, test for Cryptococcus and Koch's bacilli). The diagnosis of meningitis was considered on a cytology of $>5$ cells $/ \mathrm{mm}^{3}$, protein $>0.45 \mathrm{~g} / \mathrm{l}$ and a favorable response to presumptive treatment. Other laboratory tests included a full blood count, CRP, serology for syphilis, CD4 T lymphocytes count. Tuberculous meningitis was diagnosed in this study as probable based of a lymphocytic pleocytosis with low glucose level, high protein level $(>1.50 \mathrm{~g} / \mathrm{l})$ and low chlore level, the absence of other bacteria or fungi in the CSF and the history of close contact with patients suffering from tuberculosis in the last three months, sweating at night and/or day, weight loss and meningeal signs associated with fever. Cryptococcal meningitis, purulent meningitis or syphilis was suspected in the presence of a febrile meningeal syndrome, the identification of a spe- cific organism in the CSF, a positive syphilis serology or the presence of soluble antigens in CSF.

The diagnosis of nonspecific viral encephalitis was suspected in the presence of clinical signs associated with signs of meningeal irritation, fever, behavior changes, loss of consciousness as well as lymphocytosis and a moderately high level of proteins in the CSF. The methods used did not permit viral isolation. PCR for tuberculosis, cryptococcosis, herpes simplex, varicella zoster, and cytomegalovirus was not performed.

A metabolic etiology of seizures was suspected when there was an abnormality in the blood electrolytes levels, without any other associated pathology and the only treatment was correction of the abnormality.

Statistical analysis was performed with commercial software SPSS 16.0 for windows. Interval and ordinal scaled data were compared using the Mann-Witney-U test and nominal (scaled data with $\mathrm{X}^{2}$-test or Fisher's exact test. Significance level was set at $p<0.05$ for two) tailed test.

\section{Results}

We consulted the files of $1572 \mathrm{HIV}$-infected patients in the Neurology, Infectious Disease and Day Hospital unit which is the national reference center for the care of people living with HIV. 150 patients $(9.5 \%)$ had seizures; Out of the 150 patients, 39 patients $(26 \%)$ were recruited in the prospective phase and 111 patients $(74 \%)$ in the retrospective phase. There were 943 hospitalizations during the study period, 196 hospitalizations were due to HIV infection (20.8\%) of which 61 (31\%) had seizures.

\subsection{Epidemiological Characteristics}

Gender: We recruited a total of 150 patients including 65 men (43.3\%) and 85 women (56.7\%). Age: Our patients were aged 19 to 75 years with an average age of $38.7 \pm 9.9$ years. The age groups most represented were 40 - 44 years $(24.2 \%)$ and $30-34$ years $(20.8 \%)$. Fourty nine percent of patients were between 30 and 44 years and $72.6 \%$ between 25 and 54 years. Marital Status: Fifty two percent of our patients were single, $35.3 \%$ married and $8 \%$ widows.

\subsection{Clinical Features}

The duration of knowledge of HIV status: Fifty patients $(33 \%)$ had their HIV status during hospitalization, 26 patients 3 months before the admission and 18 patients one week before. The time delay between the diagnosis of HIV infection and the consultation is shown on Figure 1.

Duration of antiretroviral treatment: One hundred and twenty two patients $(81.3 \%)$ were not on antiretrovi- 
ral drugs (ARVs) on arrival at the hospital. Only 28 patients were on ARVs, including 7 patients taking their medications for a year, 10 patients for 3 months and nearly two thirds $(68 \%)$ were taking ARVs for less than 3 months.

Reason for consultation. The distributions of patients according to the main complain on admission is shown on Table 1.

Seizures type: generalized seizures were found in 99 patients $(66 \%)$ and partial seizures in 51 patients (34\%). A status epilepticus was found in 27 patients $(18 \%)$.

Focal neurological deficit: Sixty two patients had hemiplegia, nine patients had peripheral facial paralysis, two patients presented with paraplegia and quadriplegia in two patients. 48 patients (32\%) had involvement of higher mental functions with disorientation in time and space for 33 cases, aphasia in 21 cases and agnosia in 8 cases.

Fever was found during hospitalization in 106 patients $(70.6 \%)$ while the temperature was normal in 44 patients $(29.4 \%)$. Meningeal irritation was observed in 56 patients $(37.3 \%)$ with neck stiffness and the presence of a Kernig sign and/or Brudzinski sign.

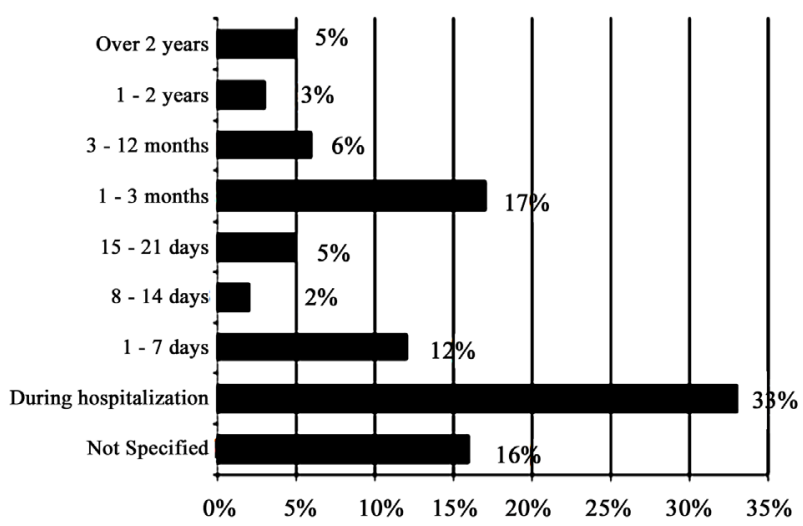

Figure 1. Distribution by period of HIV knowledge.

Table 1. Distribution of patients according to the reasons for consultation.

\begin{tabular}{ccc}
\hline Reasons for consultation & Cases & Percentage (\%) \\
\hline Seizures & 82 & 54.66 \\
Fever & 56 & 37.33 \\
Headache & 53 & 35.33 \\
Impaired awareness & 30 & 20 \\
Neurological deficit & 26 & 17.33 \\
Behavioral disorder & 22 & 14.66 \\
Digestive disorders & 13 & 8.66 \\
Others & 25 & 16.66 \\
\hline
\end{tabular}

\subsection{Paraclinical Investigation}

HIV serology: All our patients had a positive antiretroviral serology by ELISA confirmed by Western Blot. HIV-type 1 was found on $87 \%$ of cases and $13 \%$ of patients had HIV-1 and HIV-2 coinfection.

The CT scan was performed in 55 patients $(36.6 \%)$, and revealed 29 images of abscesses, 8 patients with cortical atrophy, 7 cases of meningoencephalitis, 2 cases of ischemic stroke and 7 normal examinations.

The electroencephalogram (EEG) was carried out in 24 patients of which only 18 (75\%) had epileptic abnormalities (slow spike waves, polyspikes waves, rhythmic slow waves), normal in 6 patients (25\%).

The CD4 lymphocyte counts: this was done only in 81 patients $(54 \%)$. In these patients, the number of TCD4 ranged from 1 to $464 / \mu 1$ with an average of $65.4 / \mu 1.49$ patients had a TCD4 count less than $100 / \mu 1$ and 9 patients a CD4 more than $250 / \mu 1$.

Serum electrolytes: Only 17 patients did the test, the serum electrolytes were normal in 12 of them $(70.5 \%)$ and abnormalities found in 5 others. All the five had hyponatremia $\left(\mathrm{Na}^{+}<135 \mathrm{mmol} / \mathrm{l}\right)$ and two had hypomagnesemia $\left(\mathrm{Mg}^{2+}<18 \mathrm{mg} / \mathrm{l}\right)$. These disorders were considered the etiology of seizures in those patients.

CSF analysis: In our study, 54 patients $(36 \%)$ underwent a CSF analysis, 36 (66.7\%) had a clear CSF, nine $(16.7 \%)$ were turbid and 9 hematic. Cryptococcus neoformans was found in 23 patients, sterile liquid in 19 cases and probable tuberculous meningitis in 6 patients.

Etiologies of seizures: Cerebral toxoplasmosis was the most common cause, found in 45 patients $(30 \%), 23$ cases of Cryptococcus meningitis, 8 cases of tuberculous meningitis (see Table 2).

Table 2. Distribution of patients depending on the etiology of seizures.

\begin{tabular}{lcc}
\hline \multicolumn{1}{c}{ Etiologies of seizures } & Cases & Percentage (\%) \\
\hline Cerebral toxoplasmosis & 45 & 30 \\
Undetermined causes & 42 & 28 \\
Cryptococcal meningitis & 23 & 15.3 \\
Probable tuberculous meningitis & 8 & 5.3 \\
Probable lymphoma & 7 & 4.6 \\
Bacterial meningitis & 5 & 3.3 \\
Viral encephalitis & 5 & 3.3 \\
Metabolic imbalance & 5 & 3.3 \\
Ischemic stroke & 4 & 2.6 \\
Severe anemia & 3 & 2 \\
HIV dementia & 2 & 1.3 \\
Syphilitic meningitis & 1 & 0.6 \\
Total & 150 & 100 \\
\hline
\end{tabular}


Cerebral toxoplasmosis: This diagnosis was made in 45 patients including 27 females $(60 \%)$ and 18 males $(40 \%)$. The age range of $40-44$ years was the most represented $(31 \%)$. Among patients in whom the diagnosis of cerebral toxoplasmosis was retained, $60 \%$ had focal seizures, and $40 \%$ had generalized seizures. Motor deficit in the form of hemiparesis or hemiplegia was observed in 44 patients $(97.7 \%) .20$ patients of them did a brain scan which revealed images of brain abscess in all and suggestive of cerebral toxoplasmosis. The average CD4 T lymphocytes count was $49.8 \pm 122.6 / \mu 1$. In addition to the antiepileptic treatment, 14 patients $(31 \%)$ were treated with the combination Sulfamethoxazole and Trimethoprim and 41 patients $(69 \%)$ by the combination pyrimethaminesulfadiazine-folinate. The evolution was marked by a recovery in 43 patients and death in 2 patients who had discontinued treatment for financial reasons.

Cryptococcal meningitis: This diagnosis was found in 23 patients including 16 females $(69.5 \%)$ and 7 males $(30.5 \%)$. The age group most represented was that of 40 44 years (39\%). 82.6\% had generalized seizures and 17.4 partial seizures \%. 22 patients $(95.6 \%)$ had meningeal sign on admission and 4 patients had a sign of raised intracranial pressure. The later did brain scan which revealed in all four patients diffuse cerebral edema with contrast enhancement at the convexity and cortical folds. CSF examination with Indian ink revealed in all these patients Cryptococcus neoformans. The average CD4 count was $51 \pm 109 / \mu 1$. All were treated with fluconazole with an improvement in $46.7 \%$ of patients, while $53.3 \%$ died.

Tuberculous meningoencephalitis: This diagnosis was made in 8 patients including 3 females and 5 males. Their average age was $43.8 \pm 8.24$ years. Seventy five percent had generalized seizures and $25 \%$ focal seizures. All these patients had signs of meningeal irritation while 5 had a focal deficit in muscle strength in the form of hemiparesis, peripheral facial palsy in 3 patients and 2 with signs of raised intracranial pressure. Half of these patients were able to do a CT-scan revealing parietal abscesses in 3 of them, this examination was normal for the fourth patient. Examination of CSF after staining with Ziehl Nelson and culture on Lowenstein did not demonstrated the Koch Bacillus in our patients, but all had elevated lymphocytic cells, elevated protein, lower glucose and 3 with lower chlorine. Their mean CD4 count was $44.09 \pm 2.30 / 1$. The evolution was marked by a recovery in 4 patients $(50 \%)$, death in one patient and three others were lost to follow up.

Associated pathologies in patients who died: $17(32 \%)$ patients died on first day of hospitalization, 28 (53\%) in the first week ( $2-7$ days) and $8(15 \%)$ after more than one week of hospitalization. The different causes of death are detailed in Table 3 and related disorders in patients who died are presented in Figure 2.

\section{Discussion}

\subsection{Characteristics of the Study Population}

HIV infection affects the whole population and especially the age group in the period of sexual activity. The average age in our study was 38.7 years and ranged from 19 to 75 years. A similar mean age of 39.2 years was found by Kuate et al. [8], not very different from that found by Modi et al. [9] in a South African study whith 33.5 years and that of Millogo et al. [10] in Burkina Faso who was 35 years. In many studies, a high proportion of seizures associated with HIV infection was found in subjects between 30 and 40 years [11-14].

Infection of people at older ages is possible. We have found 75 years as maximum age in our study while Kuate et al. [8] found 63 years, and Millogo et al. [10] 71 years as maximum age. This allows the clinician to seek HIV status to all patients with seizures regardless of age, before any clinical features suggestive of immunosuppression.

\subsection{Proportion of Seizures in HIV Infection}

Most data on new onset seizures in patients infected with HIV are from hospital series and reported seizures in $2 \%$ - $20 \%$ of people living with HIV $[5,6]$.

Table 3. Distribution of patients who died according to etiology.

\begin{tabular}{lcc}
\hline \multicolumn{1}{c}{ Etiologies } & Deaths & Percentage (\%) \\
\hline Cryptococcal meningitis & 12 & 22.64 \\
Probable cerebral lymphoma & 7 & 13.20 \\
Cerebral toxoplasmosis & 2 & 3.77 \\
Tuberculous meningitis & 1 & 1.88 \\
Undetermined causes & 31 & 58.49 \\
Total & 53 & 100 \\
\hline
\end{tabular}

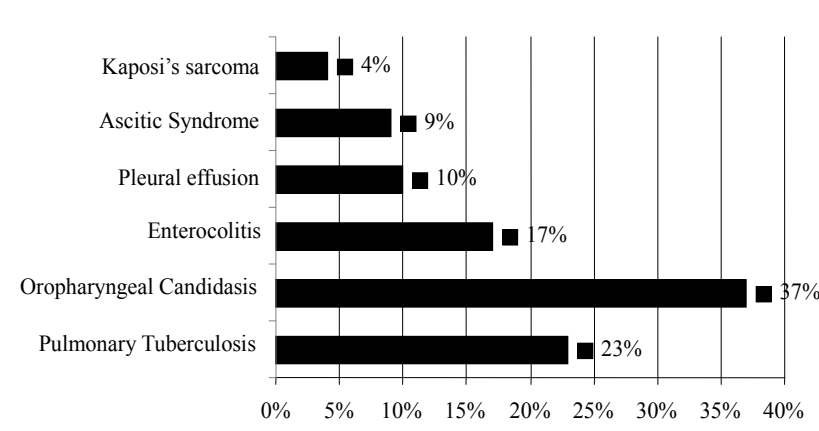

Figure 2. Distribution of associated pathologies in patients who died. 
In our series, 150 cases of seizures of recent onset $(9.5 \%)$ were observed in 1572 records of patients with HIV infection followed at the Yaoundé Central Hospital (Department of Neurology, Department of infectious diseases, Intensive care unit, and day care Hospital). If we considered only patients admitted in the neurology department, out of $196 \mathrm{HIV}$ infected patients, 61 (31\%) had seizures. The very high frequency of seizures in our cohort of patients compared to other studies could be related to selection bias, the latter being referred to the only neurology department mainly for neurological complications. On the other hand, in Cameroon as in other developing countries, an increased incidence of opportunistic infections of the CNS appears to be the cause of this high proportion of seizures. As part of the great pandemic of HIV, particularly affecting developing countries, many of these seizures are seen as symptomatic of brain damage caused by opportunistic infections [15].

\subsection{Semiology of Seizures}

In our study, $66 \%$ of patients had generalized seizures, and 34\% partial seizures. Chadha et al. in India [5] found $65.2 \%$ of patients with generalized seizures and focal seizures for 34.8\%. Bartholomei et al. in France [7] found $71 \%$ of generalized seizures and partial seizures in $29 \%$. Kuate et al. [8] in a study conducted in Cameroon in 30 patients reported a predominance of partial seizures $(80 \%)$. $63 \%$ of Satishchandra and Sinha's patients [14] also had generalized seizures. Focal seizures are less common than generalized seizures and are not necessarily related to focal expansive lesions. Partial seizures secondarily generalized or not can be observed in diffuse brain pathologies such as meningitis or HIV encephalopathy [11]. The high proportion of generalized seizures can be explained by a very rapid secondary generalization or by the lack of experience and difficulties of the care givers or general practitioners to give a precise description of the seizurs and especially their focal origin [14]. These results reveal the diversity of seizure semiology in neurological lesions due to HIV, but these differences could also be explained by the number of focal lesions found on brain $\mathrm{CT}$ in the different studies, because according to Pascual-Sedano [4] partial seizures suggest the possibility of an intracranial expansive process.

A status epilepticus was found in $27(18 \%)$ of our patients. A status epilepticus, most often associated with poor prognosis has been reported in a percentage between $8 \%$ and $18 \%$ of cases, in several studies $[6,9,16]$. The high frequency of status epilepticus suggests that the brain infected with HIV has a low seizure threshold related to relative imbalance of excitatory and inhibitory neurotransmitters and neurotoxicity of other substances in brain that may predispose to seizures [15].

We have found $54.6 \%$ of patients consulting for seizures, revealing HIV infection in $29.3 \%$ of them. Bartholomei et al. [7] in their study found $2 \%$ of HIV patients in whom seizures revealed the infection. Holtzman et al. [11] in a study in the USA found that seizures revealed infection in $18 \%$ of patients. This reflects the delayed arrival of patients in hospitals in our region and even the relative stigma of epilepsy and HIV infection in Africa in general.

\subsection{HIV Serology}

The diagnosis of HIV infection involves a specific test to distinguish the viral type. HIV-1 is the main type found in our study with $89.7 \%$, Millogo et al. [10] in their study found $100 \%$ of patients with HIV-1. Given that the viral type was not specified in all patients $(35.3 \%)$, the fact that HIV-2 was always associated with type 1 in $6 \%$ of our patients, we cannot confirm the involvement of HIV1 alone in lesions of the central nervous system (CNS) as described in the literature [17].

$50.6 \%$ of patients already knew their HIV status before their arrival at the hospital and $18.7 \%$ were already on ART. None of the patients reported by Millogo et al. [10] were on ART, although all were HIV-infected. These data confirm that a very high proportion of patients infected with HIV in our study are not yet on ARVs, which explains the high proportion of opportunistic infections associated with severe immunosuppression.

\subsection{CD4-Lymphocyte Count}

The number of CD4 T lymphocytes is a parameter which reflects the degree of immunosuppression in patients infected with HIV. In our study the average rate of CD4 $\mathrm{T}$ lymphocytecells was estimated at 105.4/ $\mu$ l. Modi et al. [9] reported that the majority of South African patients had CD4 cell counts below 200, Millogo et al. [10] reported that $74 \%$ of patients had fewer than $200 / \mu 1 \mathrm{CD} 4$ cells and an average of 236/ $\mu$ l. This low CD4 level reveals the profound immunosuppression of our patients, confirming what is described in the literature: "more the number of CD4 T lymphocytes are lower, more frequent are opportunistic infections of the central nervous system" [3].

\subsection{The Brain CT Scan}

Brain CT-scan in our study, revealed $52.7 \%$ of patients with intracranial mass lesion. Kuate et al. [8] had found $60 \%$ of patients with intracranial masses and Modi et al. [9] $53.3 \%$. These results show similarly high prevalence of brain abscesses in our setting where few patients are 
on ARVs. It remains possible that small or diffuse lesions were not recognized because they are difficult to recognize on the CT-scan and better seen on MRI examination that was not available in Cameroon at the time of the study. This raises the problem of differential diagnosis in immunocompromised patients with seizures where cerebral toxoplasmosis is often evoked in the absence of imaging. Brain MRI is an exploration of choice for comprehensive assessment of brain damage in immunocompromised patients with HIV.

\subsection{CSF Analysis}

In our study, a CSF was collected in 56 patients and analysis showed abnormal result in $64.8 \%$ of them. In the study by Modi et al. [9], 32 patients with space occupying lesion, had abnormal CSF in 78\% revealing a pleocytosis $(9 \%)$, elevated protein $(59 \%)$, cysticercosis $(21 \%)$, tuberculosis $(3 \%)$, and syphilis (3\%). For 13 patients with a meningitis, all had abnormal CSF with elevated protein $(92 \%)$, pleocytosis $(46 \%)$, cryptococcal antigen (31\%), and syphilis (15\%). These results demonstrate the high tropism of HIV and other germs to the central nervous system.

\subsection{Electroencephalogramme (EEG)}

In a study by Kellinghaus et al. [13] $27 \%$ of patients had a normal EEG, 17\% generalized slow waves, $6 \%$ a combination of focal and generalized slow waves, localized slow waves in $22 \%$ of patients, and only one had focal epileptic discharges. This examination was performed in few patients $(16 \%)$ in our series, revealing paroxysmal epileptic discharges in $1 / 3$ of them. $75 \%$ of patients had an abnormal EEG. Meanwhile Parisi et al. [18] found that $67 \%$ of immunocompromised HIV patients have an abnormal EEG of which $37 \%$ were probably due to opportunistic infections of the central nervous system.

In the literature, many EEG abnormalities are described in patients infected with HIV and who have had one or more epileptic seizures: focal or generalized slow waves, localized epileptic discharges and periodic lateralized epileptic discharges (PLEDs) [19,20]. A specifically altered EEG can also be observed in patients infected with HIV but asymptomatic [21].

Slow and repetitive graphic elements described in advanced stage of the disease are noted early in infection. During evolution, there is a reactivation and spatialisation defect as well as decrease in the amplitude of background activity [22]. One can also observe a slowing or decrease in the amplitudes of the posterior regions. At advanced stages of the disease often characterized by a demented state, slow biphasic, pseudo periodic waves predominant on the anterior [23]. Our results are consis- tent with what is described in the literature, but few African studies have investigated it.

\subsection{Etiologies of Seizures}

Cerebral toxoplasmosis: Cerebral toxoplasmosis is the most common cerebral mass lesion in patients with AIDS $[6,10]$. It was the etiology of seizures in $30 \%$ of our patients. Kuate et al. [8] in a similar study with only 30 patients in Cameroon found $56.7 \%$. According to Njamnshi et al. [24], cerebral toxoplasmosis represents $17.5 \%$ to $50 \%$ of neurological complications of HIV in Cameroon. Holtzmann et al. [11] reported $22 \%$ in the USA, Modi et al. [9] a 4\% rate in South Africa, Millogo et al. [10] 65\% in Burkina Faso. The difference with the South African study was due to the high prevalence of tuberculosis in that country. The majority of US patients was on ARVs and was therefore less vulnerable to opportunistic infections including toxoplasmosis while any Burkinabe patients were on ART. More over in the latter, no brain imaging was available to eliminate other differential diagnoses. Our results are consistent with the literature, confirming that cerebral toxoplasmosis is the first etiology of seizures on HIV-positive patients $[5,6]$.

Patients with cerebral toxoplasmosis had a mean CD4 count of $49.8 / \mu 1$. Millogo et al. [10] found $55.81 \%$ with a CD4 count below 200/ $\mu$ l. This confirms that cerebral toxoplasmosis is an opportunistic infection occurring at very advanced stages of immunosuppression.

Concerning semiology of seizures, $40 \%$ of patients had generalized seizures, and $60 \%$ focal seizures, Millogo et al. [10] in Burkina Faso found 64\% with focal seizures, with or wtihout secondarily generalization. Seizures of recent onset in a patient infected with HIV are usually due to raised intracranial process, foremost among which is cerebral toxoplasmosis resulting most often in partial seizures [18].

Cryptococcal meningitis: Its frequency varies from 5.5 to $22 \%$ in HIV infection [25]. It was found in $15.3 \%$ of cases in our study while Millogo et al. [10] reported $16 \%$ in Burkina Faso, Modi et al. [9] 8\% of cryptococcal meningitis. Our results are similar to those in the literature and confirm the high prevalence of this infection in our region. Indeed according to Cabre [26] the prevalence of this infection is high in sub-Saharan Africa and can affect up to $30 \%$ HIV infected patients. This high prevalence can be explained by the presence of Cryptococcus in the environment in which AIDS patients live.

In this study, meningeal cryptococcosis is associated with high mortality rates $(53.3 \%)$ as in many other studies [10,27]. Mbuagbaw et al. [25] in a study at the Yaounde University Hospital found $42.2 \%$ of deaths from cryptococcal meningitis. The long duration of symptoms and poor adherence to treatment are poor prognostic factors 
in this study, these factors being also responsible for the deaths in our study. According to Mwamba et al. [28], cryptococcosis is the most deadly infection of the nervous system in the context of immunosuppression. In a retrospective study of 230 Zambian patients suffering from this condition, the authors obtained $100 \%$ mortality at 7 weeks in the group receiving no treatment, and 6 months in the group receiving antifungal therapy [28]. Fluconazole is now given free to patients in Cameroon and this could help reducing mortality from this disease.

Causes not determined: We did not find the cause of seizures in $28 \%$ of our patients. Modi et al. [9] in South Africa had $25 \%$ of undetermined causes; Chadha et al. [5] in India in a similar study had $30.4 \%$ of unknown causes of seizures and Pesola et al. [29] in the USA 30.7\%. In our environment this high rate is due to the lack of thorough investigation and premature death of patients due to their late arrival in hospital. Several authors like Wong [6] and Holtzman [11] suggest that HIV itself is the cause of seizures in this subgroup. A long term follow up of this group could give us more information.

The antiepileptic treatment: The recurrence of seizures is common in patients infected with HIV. A longterm antiepileptic treatment should therefore be considered even after a single seizure. In our study, $29.3 \%$ of patients were treated with carbamazepine, $26.7 \%$ with phenobarbital and $37.3 \%$ had no antiepileptic drug. Brew et al. [30] suggested that Phenytoin is the most appropriate medication for epileptic seizures in the HIV-positive patient. In a study on the interaction between antiretroviral and anti-epileptic drugs; Jennings et al. [31] estimate that the sodium Valproate is contra-indicated in these subjects as it leads to increased viral replication. More recent studies have shown that valproate inhibits an enzyme in chromatin remodeling, responsible for maintaining the infection of CD4 cells by HIV [32]. However, a pilot study reveals that in three out of four patients, there is a significant reduction of on $\mathrm{T}$ cells infection in patients taking concomitant ART and valproate [33]. In a recent study, Mehling et al. [34] evaluate the interactions between ARV drugs and antiepileptic drugs in patients with HIV, and recommended as first-line treatment for seizures: valproate, gabapentin, oxcarbazepine, pregabalin and and leveticacetam. These authors recommended as second line: lamotrigine, topiramate, tiagabine and zonisamide. Phenobarbital, phenytoin, and carbamazepine should be avoided and benzodiazepines keeped for emergencies as well as short-term treatments due to their short duration of action and their sedative effect [34].

\section{Conclusion}

The study of seizures of recent onset in patients with HIV infection allows us to draw the following conclu- sions: 1) the prevalence of seizures in these patients is $9.5 \%$; 2) the seizure was the initial manifestation in $55.6 \%$ of cases revealing HIV infection in $29.3 \%$ of them; $3)$ generalized seizures were more frequent $(66 \%)$ and status epilepticus was found in 27 patients $(18 \%) ; 4)$ cerebral toxoplasmosis was the most common etiology (30\%) followed by cryptococcal meningitis, tuberculous meningoencephalitis, and $28 \%$ of patients had no specific aetiology.

\section{REFERENCES}

[1] S. A Ogun, F. Ojini, N. Okubadejo, M. Danesi, K. Kolabo, B. Osalusi and B. Brian, "Les Manifestations Neurologiques du VIH-SIDA: Revue de 154 cas dans un Centre Hospitalier Nigérian," African Journal of Neurological Sciences, Vol. 22, No. 1, 2005, pp. 29-36.

[2] A. I. Bhigjee, "Seizures in HIV/AIDS: A Southern African Perspective," Acta Neurologica Scandinavica, Vol. 112, No. S181, 2005, pp. 4-7. doi:10.1111/j.1600-0404.2005.00500.x

[3] S. Attili, Venkata, A. Kumar, V. P. Singh, D. V. Varma, M. Rai and S. Sundar, "Manifestations Neurologiques chez les Patients Infectés par le VIH dans la Région de Varanasi (Inde)," African Journal of Neurological Sciences, Vol. 25, No. 1, 2006, pp. 33-40.

[4] B. Pascual-Sedano, A. Iranzo, J. Marti-Fabregas, P. Domingo, A. Escartin, M. Fuster, J. L. Barrio and M. A. Sambeat, "Prospective Study of New Onset-Seizures in Patients with Human Immunodeficiency Virus Infection: Etiologic and Clinical Aspects," Archives of Neurology, Vol. 56, No. 5, 1999, pp. 609-612.

[5] D. S. Chadha, A. Handa, S. K. Sharma, P. Varadarajulu and A. P. Singh, "Seizures in Patients with Human Immunodeficiency Virus Infection," Journal of the Association of Physicians of India, Vol. 48, No. 6, 2000, pp. 573576.

[6] M. C. Wong, N. D. Suite and D. R. Labar, "Seizures in Human Immunodeficiency Virus Infection," Archives of Neurology, Vol. 47, No. 6, 1990, pp. 640-642.

[7] F. Bartholomei, P. Pellegrino, C. Dhiver, R. Quilichini, J. A. Gastaut and J. L. Gastaut, "Epilepsy Seizures in HIV Infection: 52 Cases," La Presse Médicale, Vol. 20, No. 42, 1991, pp. 21-35.

[8] C. Kuate, S. Mbahe, P. Ngang, F. Yepnjio, F. Djeutcheu and A. K. Njamnshi, "Epilepsie et Infection à VIH à l'Hôpital Central de Yaoundé," Revue Neurologique, Vol. 162S, 2007, p. 118.

[9] G. Modi, M. Modi, I. Martinus and D. Saffer, "NewOnset Seizures Associated with HIV Infection," Neurology, Vol. 55, No. 10, 2000, pp. 1558-1560. doi:10.1212/WNL.55.10.1558

[10] A. Millogo, D. Lankoandé, I. Yaméogo, A. A. Yaméogo, A. Sawadogo and A. B. Sawadogo, "New-Onset Seizures in Patients with Immunodeficiency Infection in BoboDioulasso Hospital (Burkina Faso)," Bulletin de la Société de Pathologie Exotique, Vol. 97, No. 4, 2004, pp. 
268-270.

[11] D. M. Holtzman, D. A. Kaku and Y. T. So, "New-Onset Seizures Associated with Human Immunodeficiency Virus Infection: Causation and Clinical Features in 100 Cases," American Journal of Medicine Vol. 87, No. 2, 1989, pp. 173-177. doi:10.1016/S0002-9343(89)80693-X

[12] W. Van Paesschen, C. Bodian and H. Maker, "Metabolic Abnormalities and New-Onset Seizures in Human Immunodeficiency Virus-Seropositive Patients," Epilepsia, Vol. 36, No. 2, 1995, pp. 146-150.

[13] C. Kellinghaus, C. Engbring, S. Kovac, G. Möddel, F. Boesebeck, M. Fischera, K. Anneken, K. Klönne, D. Reichelt, S. Evers and I. W. Husstedt, "Frequency of Seizures and Epilepsy in Neurological HIV-Infected Patients," Seizure, Vol. 17, No. 1, 2008, pp. 27-33. doi:10.1016/j.seizure.2007.05.017

[14] P. Satishchandra and S. Sinha, "Seizures in HIV-Seropositive Individuals: NIMHANS Experience and Review," Epilepsia, Vol. 49, Suppl. 6, 2008, pp. 33-41. doi:10.1111/j.1528-1167.2008.01754.x

[15] R. K. Garg, "HIV Infection and Seizures," Postgraduate Medical Journal, Vol. 75, No. 885, 1999, pp. 387-390.

[16] M. Modi, A. Mochan and G. Modi, "New Onset Seizures in HIV-Seizure Semiology, CD4 Counts, and Viral Loads," Epilepsia, Vol. 50, No. 5, 2009, pp. 1266-1269.

[17] C. Lacroix, "Manifestations Neurologiques du VIH," Elsevier, Paris, 1999, 11 p.

[18] A. Parisi, M. Strosseli, A. Pan, R. Maserati and L. Minoli, "HIV-Related Encephalitis Presenting as Convulsant Disease," Clinical EEG (Electroencephalography), Vol. 22, No. 1, 1991, pp. 1-4.

[19] R. F. Cury, L. Wichert-Ana, A. C. Sakamoto and R. M. Fernandes, "Focal Non-Convulsive Status Epilepticus Associated to PLEDs and Intense Focal Hyperaemia in an AIDS Patient," Seizure, Vol. 13, No. 5, 2004, pp. 358-361. doi:10.1016/j.seizure.2003.07.001

[20] G. Ozkaya, A. Kurne, S. Unal, K. K. Oguz, R. Karabudak and S. Saygi, "Aphasic Status Epilepticus with Periodic Lateralized Epileptiform Discharges in a Bilingual Patient as a Presenting Sign of AIDS Toxoplasmosis Complex," Epilepsy \& Behavior, Vol. 9, No. 1, 2006, pp. 193-196. doi:10.1016/j.yebeh.2006.04.005

[21] D. H. Gabuzda, S. R. Levy and K. H. Chiappa, "Electroencephalography in AIDS and AIDS-Related Complex," Clinical EEG (Electroencephalography), Vol. 19, No. 1, 1988, pp. 1-6.

[22] C. L. Harden, M. Daras, A. J. Tuchman and B. S. Koppel, "Low Amplitude EEG's in Demented AIDS Patients," Electroencephalography and Clinical Neurophysiology, Vol. 87, No. 1, 1993, pp. 54-56. doi:10.1016/0013-4694(93)90174-T

[23] A. Beaumanoir and A. Nahory, "L'EEG dans l'Infection par le VIH," Neurophysiologie Clinique, Vol. 22, No. 5, 1992, pp. 355-369. doi:10.1016/S0987-7053(05)80094-2
[24] A. K. Njamnshi, V. P. Djientcheu, A. Bissek, P. OngoloZogo, N. Mapoure, F. Yepnjio, V. Sini, L. Dongmo, L. Kaptué and W. Muna, "Epidemiologocal and Aetiological Features of Nervous System HIV-AIDS in Yaounde: A Preliminary Study," Health Sciences and Disease, Vol. 3, No. 2003, pp. 18-28.

[25] J. Mbuagbaw, Biholong and A. K. Njamnshi, "La Cryptococcose Neuro-Meningée et l'Infection au VIH dans le Service de Médecine du Centre Hospitalier et Universitaire de Yaoundé, Cameroun," African Journal of Neurological Sciences, Vol. 25, No. 2, 2006, pp. 13-20.

[26] P. Cabre, D. Smadja and A. Cabié, "Newton C.R.J. HTLV-1 and HIV Infections in the Central Nervous System in Tropical Areas," Journal of Neurology, Neurosurgery \& Psychiatry, Vol. 68, No. 5, 2000, pp. 550-557. doi:10.1136/jnnp.68.5.550

[27] P. Desmet, K. D. Kayembe and C. De Vroey, "The Value of Cryptococcal Serum Antigen Screening among HIVPositive/AIDS Patients in Kinshasa, Zaire," AIDS, Vol. 3, No. 2, 1989, pp. 77-78. doi:10.1097/00002030-198902000-00004

[28] P. Mwamba, J. Mwansa and C. Chintu, "Clinical Presentation Natural History and Cumulative Death Rates of 230 Adults with Primary Cryptococcal Meningitis in Zambian AIDS Patients Treated under Local Conditions," Postgraduate Medical Journal, Vol. 77, No. 914, 2001, pp. 769-773. doi:10.1136/pmj.77.914.769

[29] G. R. Pesola and R. E. Westfal, "New-Onset Generalised Seizures in Patients with AIDS Presenting to an Emergency Department," Academic Emergency Medicine, Vol. 5, No. 9, 1998, pp. 905-911.

[30] B. J. Brew, "Medical Management of AIDS Patients. Central and Peripheral Nervous System Abnormalities," Medical Clinics of North America, Vol. 76, No. 1, 1992, pp. 63-81.

[31] H. R Jennings and F. Romanelli, "The Use of Valproic Acid in HIV-Positive Patients," Annals of Pharmacotherapy, Vol. 33, No. 10, 1999, pp. 1113-1116. doi:10.1345/aph.19014

[32] L. Ylisastigui, N. Archin, G. Lehrman, R. J. Bosch and D. M. Margolis, "Coaxing Human Immunodeficiency Virus Type 1 from Resting CD4+ T Cells: Can the Reservoir of HIV Be Purged," AIDS, Vol. 18, No. 8, 2004, pp. 11011108. doi:10.1097/00002030-200405210-00003

[33] G. Lehrman, I. B. Hogue, S. Palmer, C. Jennings, C. A. Spina and A. Wiegand, "Depletion of Latent HIV-Infection in Vivo: A Proof-of-Concept Study," Lancet, Vol. 366, No. 9485, 2005, pp. 549-555. doi:10.1016/S0140-6736(05)67098-5

[34] M. Mehling, H. Drechsler, J. Kuhle, M. Hardmeier, R. Doerries, S. Ruegg and A. Gass, "Adaptation of Antiretroviral Therapy in Human Immunodeficiency Virus Infection with Central Nervous System Involvement," Journal of Neurovirology, Vol. 14, No. 1, 2008, pp. 78-84. doi: $10.1080 / 13550280701793940$ 